\title{
A FURTHER GENERALIZATION OF THE CATALAN NUMBERS AND ITS EXPLICIT FORMULA AND INTEGRAL REPRESENTATION
}

\author{
WEN-HUI LI, FENG QI*, OMRAN KOUBA, AND ISSAM KADDOURA
}

\begin{abstract}
In the paper, motivated by the generating function of the Catalan numbers in combinatorial number theory and with the aid of Cauchy's integral formula in complex analysis, the authors generalize the Catalan numbers and its generating function, establish an explicit formula and an integral representation for the generalization of the Catalan numbers and corresponding generating function, and derive several integral formulas and combinatorial identities.
\end{abstract}

\section{Contents}

1. Backgrounds and motivations 1

2. $\quad$ An explicit formula for $\mathfrak{C}_{n}(a, b ; p)$

3. An integral representation of the generating function $G_{a, b ; p}(z)$

\begin{tabular}{ll|l} 
4. An integral representation for $\mathfrak{C}_{n}(a, b ; p)$ & 8
\end{tabular}

5. Two integral formulas $\quad 9$

\begin{tabular}{lll}
\hline 6. & An elementary computation of the integral in (5.2) & 13
\end{tabular}

\begin{tabular}{ll}
\hline 7. More remarks & 17
\end{tabular}

$\begin{array}{ll}\text { References } & 18\end{array}$

\section{BACKGROUNDS AND MOTIVATIONS}

This paper is a continuation of the article [39.

The Catalan numbers

$$
C_{n}=\frac{1}{n+1}\left(\begin{array}{c}
2 n \\
n
\end{array}\right)=\frac{4^{n} \Gamma(n+1 / 2)}{\sqrt{\pi} \Gamma(n+2)}
$$

form a sequence of integers (see [10, 11, 53]), can be interpreted combinatorially (see [6, 16, 50]), date back to the year 1730 (see [17, 18, 21), and can be generated (see [24, 50, 54]) by

$$
G(x)=\frac{2}{1+\sqrt{1-4 x}}=\sum_{n=0}^{\infty} C_{n} x^{n},
$$

where

$$
\Gamma(z)=\int_{0}^{\infty} t^{z-1} e^{-t} \mathrm{~d} t, \quad \Re(z)>0
$$

2010 Mathematics Subject Classification. Primary 05A15; Secondary 11B75, 11B83, 26A09, 30E20, 33B15, 41A58. Key words and phrases. Catalan number; Catalan-Qi number; generating function; generalization; explicit formula; integral representation; Cauchy's integral formula; integral formula; combinatorial identity; gamma function.

${ }^{*}$ Corresponding author.

This paper was typeset using $\mathcal{A}_{\mathcal{M}} \mathcal{S}$ - $\mathrm{LAT}_{\mathrm{E}} \mathrm{X}$. 
or

$$
\Gamma(z)=\lim _{n \rightarrow \infty} \frac{n ! n^{z}}{\prod_{k=0}^{n}(z+k)}, \quad z \in \mathbb{C} \backslash\{0,-1,-2, \ldots\}
$$

is the classical Euler gamma function (see [1, Chapter 6], [25, Chapter 5], [55, Chapter 3], and [33]).

The Catalan numbers $C_{n}$ have been combinatorially generalized as the Fuss numbers (see 8] and [16, pp. 377-378]), the Fuss-Catalan numbers (see [3, 5, 7, 52 and [16, Exercise A14, p. 108]), and others (see [2, 13, 15] and [16, pp. 375-376]).

Motivated by the second expression in (1.1), several mathematicians analytically generalized the Catalan numbers $C_{n}$, the Fuss numbers, and the Fuss-Catalan numbers and investigated plenty of their properties in the papers $20,23,27,28,29,30,31,32,34,35,36,37,38,40,43$, 44, 45, 46, 47, 48, 49, 51, 57] and closely related references.

Motivated by the generating function $G(x)$ in $(1.2)$, several mathematicians generalized the Catalan numbers $C_{n}$ to $\mathcal{C}_{n}(a, b)$ in [39] by considering the generating function

$$
G_{a, b}(x)=\frac{1}{a+\sqrt{b-x}}=\sum_{k=0}^{\infty} \mathcal{C}_{n}(a, b) x^{n}
$$

for real numbers $a \geq 0$ and $b>0$. It is clear that $\mathcal{C}_{n}\left(\frac{1}{2}, \frac{1}{4}\right)=C_{n}$. The main results in [39] are as follows.

(1) The Catalan-Qi numbers of the second kind $\mathcal{C}_{n}(a, b)$ for $n \geq 0, a \geq 0$, and $b>0$ can be explicitly computed by

$$
\mathcal{C}_{n}(a, b)=\frac{1}{(2 n) ! ! b^{n+1 / 2}} \sum_{k=0}^{n}\left(\begin{array}{c}
2 n-k-1 \\
2(n-k)
\end{array}\right) \frac{k ![2(n-k)-1] ! !}{(1+a / \sqrt{b})^{k+1}},
$$

where $(-1) ! !=1$.

(2) The principal branch of the generating function $G_{a, b}(z)$ for $a \geq 0$ and $b>0$ can be represented by

$$
G_{a, b}(z)=\frac{1}{a+\sqrt{b-z}}=\frac{1}{\pi} \int_{0}^{\infty} \frac{\sqrt{t}}{a^{2}+t} \frac{1}{b+t-z} \mathrm{~d} t, \quad z \in \mathbb{C} \backslash[b, \infty) .
$$

Consequently, the Catalan-Qi numbers of the second kind $\mathcal{C}_{n}(a, b)$ for $a \geq 0$ and $b>0$ can be represented by

$$
\mathcal{C}_{n}(a, b)=\frac{1}{\pi} \int_{0}^{\infty} \frac{\sqrt{t}}{a^{2}+t} \frac{1}{(b+t)^{n+1}} \mathrm{~d} t, \quad n \geq 0 .
$$

In this paper, motivated by the generating functions in 1.2 and 1.3 , we consider the functional sequence $\mathfrak{C}_{n}(a, b ; p)$ which is defined by

$$
G_{a, b ; p}(x)=\frac{1}{a+(b-x)^{p}}=\sum_{n=0}^{\infty} \mathfrak{C}_{n}(a, b ; p) x^{n}
$$

for $a \geq 0, b>0$, and $0<p<1$. It is clear that

$$
\begin{aligned}
& G_{a, b ; 1 / 2}(x)=G_{a, b}(x), \quad G_{1 / 2,1 / 4}(x)=G(x), \quad G_{1 / 2,1 / 4 ; 1 / 2}(x)=G(x), \\
& \mathfrak{C}_{n}\left(a, b ; \frac{1}{2}\right)=\mathcal{C}_{n}(a, b), \quad \mathcal{C}_{n}\left(\frac{1}{2}, \frac{1}{4}\right)=C_{n}, \quad \mathfrak{C}_{n}\left(\frac{1}{2}, \frac{1}{4} ; \frac{1}{2}\right)=C_{n},
\end{aligned}
$$

but one can neither express $G_{a, b ; p}(x)$ in terms of $G_{a, b}(x)$ or $G(x)$ nor express $\mathfrak{C}_{n}(a, b ; p)$ in terms of $\mathcal{C}_{n}(a, b)$ or $C_{n}$. In this paper, we will establish an explicit formula for $\mathfrak{C}_{n}(a, b ; p)$, present integral representations for $G_{a, b ; p}(x)$ and $\mathfrak{C}_{n}(a, b ; p)$, and derive several integral formulas and combinatorial identities. 


\section{AN EXPLiCIT FORMULA FOR $\mathfrak{C}_{n}(a, b ; p)$}

In this section, we establish an explicit formula for $\mathfrak{C}_{n}(a, b ; p)$, from which we can derive the explicit formula 1.4 .

Theorem 2.1. Let $a \geq 0, b>0$, and $0<p<1$ be real numbers. Then the functional sequence $\mathfrak{C}_{n}(a, b ; p)$ for $n \geq 0$ can be explicitly computed by

$$
\mathfrak{C}_{n}(a, b ; p)=\frac{1}{a+b^{p}} \frac{(-1)^{n}}{b^{n} n !} \sum_{k=0}^{n} \frac{1}{\left(1+a / b^{p}\right)^{k}} \sum_{\ell=0}^{k}(-1)^{\ell}\left(\begin{array}{c}
k \\
\ell
\end{array}\right)\langle p \ell\rangle_{n},
$$

where the notation

$$
\langle\alpha\rangle_{n}=\prod_{k=0}^{n-1}(\alpha-k)= \begin{cases}\alpha(\alpha-1) \cdots(\alpha-n+1), & n \geq 1 \\ 1, & n=0\end{cases}
$$

for $\alpha \in \mathbb{C}$ is called the falling factorial.

Proof. In [6, p. 139, Theorem C], noted Faà di Bruno's formula is described by

$$
\frac{\mathrm{d}^{n}}{\mathrm{~d} x^{n}} f \circ h(x)=\sum_{k=0}^{n} f^{(k)}(h(x)) \mathrm{B}_{n, k}\left(h^{\prime}(x), h^{\prime \prime}(x), \ldots, h^{(n-k+1)}(x)\right),
$$

where $f \circ h$ denotes the composite of the $n$-time differentiable functions $f$ and $h$, and the Bell polynomials of the second kind $\mathrm{B}_{n, k}\left(x_{1}, x_{2}, \ldots, x_{n-k+1}\right)$ for $n \geq k \geq 0$ are defined (see [6. p. 134, Theorem A]) by

$$
\mathrm{B}_{n, k}\left(x_{1}, x_{2}, \ldots, x_{n-k+1}\right)=\sum_{\substack{1 \leq i \leq n-k+1 \\ \ell_{i} \in\{0\} \cup \mathbb{N} \\ \sum_{i=k}^{n-k+1} i \ell_{i}=n \\ \sum_{i=1}^{n-k+1} \ell_{i}=k}} \frac{n !}{\prod_{i=1}^{n-k+1} \ell_{i} !} \prod_{i=1}^{n-k+1}\left(\frac{x_{i}}{i !}\right)^{\ell_{i}} .
$$

Let $h=h_{p}(x)=(b-x)^{p}$ for $0<p<1$. Then $h_{p}^{(k)}(x)=(-1)^{k}\langle p\rangle_{k}(b-x)^{p-k}$ for $k \geq 0$ and, in light of the formula 2.2 with $f(x)=\frac{1}{a+x}$,

$$
\begin{aligned}
\frac{\mathrm{d}^{n} G_{a, b ; p}(x)}{\mathrm{d} x^{n}}= & \sum_{k=0}^{n} \frac{\mathrm{d}^{k}}{\mathrm{~d} h^{k}}\left(\frac{1}{a+h}\right) \mathrm{B}_{n, k}\left(h_{p}^{\prime}(x), h_{p}^{\prime \prime}(x), \ldots, h_{p}^{(n-k+1)}(x)\right) \\
= & \sum_{k=0}^{n}(-1)^{k} \frac{k !}{\left[a+h_{p}(x)\right]^{k+1}} \mathrm{~B}_{n, k}\left(h_{p}^{\prime}(x), h_{p}^{\prime \prime}(x), \ldots, h_{p}^{(n-k+1)}(x)\right) \\
= & \sum_{k=0}^{n}(-1)^{k} \frac{k !}{\left[a+(b-x)^{p}\right]^{k+1}} \mathrm{~B}_{n, k}\left(-\langle p\rangle_{1}(b-x)^{p-1},\langle p\rangle_{2}(b-x)^{p-2},\right. \\
& \left.\ldots,(-1)^{n-k+1}\langle p\rangle_{n-k+1}(b-x)^{p-(n-k+1)}\right) \\
= & \sum_{k=0}^{n}(-1)^{k} \frac{k !}{\left[a+(b-x)^{p}\right]^{k+1}}(-1)^{n}(b-x)^{k p-n} \mathrm{~B}_{n, k}\left(\langle p\rangle_{1},\langle p\rangle_{2}, \ldots,\langle p\rangle_{n-k+1}\right) \\
= & \sum_{k=0}^{n}(-1)^{k} \frac{k !}{\left[a+(b-x)^{p}\right]^{k+1}}(-1)^{n}(b-x)^{k p-n} \frac{(-1)^{k}}{k !} \sum_{\ell=0}^{k}(-1)^{\ell}\left(\begin{array}{l}
k \\
\ell
\end{array}\right)\langle p \ell\rangle_{n} \\
= & \frac{(-1)^{n}}{(b-x)^{n}} \sum_{k=0}^{n} \frac{(b-x)^{k p}}{\left[a+(b-x)^{p}\right]^{k+1}} \sum_{\ell=0}^{k}(-1)^{\ell}\left(\begin{array}{c}
k \\
\ell
\end{array}\right)\langle p \ell\rangle_{n}
\end{aligned}
$$




$$
\rightarrow \frac{(-1)^{n}}{b^{n}\left(a+b^{p}\right)} \sum_{k=0}^{n} \frac{1}{\left(1+a / b^{p}\right)^{k}} \sum_{\ell=0}^{k}(-1)^{\ell}\left(\begin{array}{l}
k \\
\ell
\end{array}\right)\langle p \ell\rangle_{n}
$$

as $x \rightarrow 0$, where we used the formula

$$
\mathrm{B}_{n, k}\left(\alpha \beta x_{1}, \alpha \beta^{2} x_{2}, \ldots, \alpha \beta^{n-k+1} x_{n-k+1}\right)=\alpha^{k} \beta^{n} \mathrm{~B}_{n, k}\left(x_{1}, x_{2}, \ldots, x_{n-k+1}\right)
$$

in [6, p. 135] and the formula

$$
\mathrm{B}_{n, k}\left(\langle\alpha\rangle_{1},\langle\alpha\rangle_{2}, \ldots,\langle\alpha\rangle_{n-k+1}\right)=\frac{(-1)^{k}}{k !} \sum_{\ell=0}^{k}(-1)^{\ell}\left(\begin{array}{c}
k \\
\ell
\end{array}\right)\langle\alpha \ell\rangle_{n}
$$

in [41, Theorem 2.1] and [42, Section 1.3] for $\alpha, \beta \in \mathbb{C}$.

The equation (1.7) means that

$$
n ! \mathfrak{C}_{n}(a, b ; p)=\lim _{x \rightarrow 0} \frac{\mathrm{d}^{n} G_{a, b ; p}(x)}{\mathrm{d} x^{n}} .
$$

Consequently, we obtain the explicit formula

$$
\mathfrak{C}_{n}(a, b ; p)=\frac{1}{n !} \lim _{x \rightarrow 0} \frac{\mathrm{d}^{n} G_{a, b ; p}(x)}{\mathrm{d} x^{n}}=\frac{(-1)^{n}}{n ! b^{n}\left(a+b^{p}\right)} \sum_{k=0}^{n} \frac{1}{\left(1+a / b^{p}\right)^{k}} \sum_{\ell=0}^{k}(-1)^{\ell}\left(\begin{array}{l}
k \\
\ell
\end{array}\right)\langle p \ell\rangle_{n} .
$$

The proof of Theorem 2.1 is complete.

Remark 2.1. When taking $p=\frac{1}{2}$ in 2.1 , we derive

$$
\mathfrak{C}_{n}\left(a, b ; \frac{1}{2}\right)=\mathcal{C}_{n}(a, b)=\frac{(-1)^{n}}{n ! b^{n}(a+\sqrt{b})} \sum_{k=0}^{n} \frac{1}{(1+a / \sqrt{b})^{k}} \sum_{\ell=0}^{k}(-1)^{\ell}\left(\begin{array}{c}
k \\
\ell
\end{array}\right)\left\langle\frac{\ell}{2}\right\rangle_{n} .
$$

Further employing the identity

$$
\sum_{\ell=0}^{k}(-1)^{\ell}\left(\begin{array}{l}
k \\
\ell
\end{array}\right)\left\langle\frac{\ell}{2}\right\rangle_{n}=(-1)^{n} \frac{k ![2(n-k)-1] ! !}{2^{n}}\left(\begin{array}{c}
2 n-k-1 \\
2(n-k)
\end{array}\right)
$$

in [41, Theorem 3.2] and [42, Section 1.5], which can be derived from comparing [2.4 for $\alpha=\frac{1}{2}$ with the identity

$$
\mathrm{B}_{n, k}((-1) ! !, 1 ! !, 3 ! !, \ldots,[2(n-k)-1] ! !)=[2(n-k)-1] ! !\left(\begin{array}{c}
2 n-k-1 \\
2(n-k)
\end{array}\right)
$$

in [45, Theorem 1.2] and [42, Section 1.5] or with the identity

$$
\mathrm{B}_{n, k}\left(\left\langle\frac{1}{2}\right\rangle_{1},\left\langle\frac{1}{2}\right\rangle_{2}, \ldots,\left\langle\frac{1}{2}\right\rangle_{n-k+1}\right)=(-1)^{n+k}[2(n-k)-1] ! !\left(\frac{1}{2}\right)^{n}\left(\begin{array}{c}
2 n-k-1 \\
2(n-k)
\end{array}\right)
$$

in [41, p. 169] and [42, Section 1.5], we recover (1.4) straightforwardly.

\section{An integral REPResentation of the Generating FUnCtion $G_{a, b ; p}(z)$}

In this section, we establish an integral representation for the principal branch of the complex generating function $G_{a, b ; p}(z)$ by virtue of Cauchy's integral formula in complex analysis.

Theorem 3.1. Let $a \geq 0, b>0$, and $0<p<1$ be real numbers. Then the principal branch of the complex function

$$
G_{a, b ; p}(z)=\frac{1}{a+(b-z)^{p}}, \quad z \in \mathbb{C} \backslash[b, \infty)
$$


can be represented by

$$
G_{a, b ; p}(z)=\frac{\sin (p \pi)}{\pi} \int_{0}^{\infty} \frac{t^{p}}{a^{2}+2 a \cos (p \pi) t^{p}+t^{2 p}} \frac{1}{b+t-z} \mathrm{~d} t, \quad \arg (z-b) \in(0,2 \pi) .
$$

Proof. In complex analysis, on the cut plane $\mathbb{C} \backslash(-\infty, 0]$, the logarithmic function $\ln z=\ln |z|+$ $i \arg z$, where $i=\sqrt{-1}$ is the imaginary unit, and the principal value $\arg z$ of the argument of $z$ satisfies $|\arg z|<\pi$.

For $a \geq 0$ and $0<p<1$, let

$$
F_{a ; p}(z)=\frac{1}{a+\exp [p \ln (-z)]}, \quad z \in \mathbb{C} \backslash[0, \infty), \quad \arg z \in(0,2 \pi) .
$$

By virtue of Cauchy's integral formula in complex analysis, for any fixed point $z_{0}=x_{0}+i y_{0} \in$ $\mathbb{C} \backslash[0, \infty)$, we have

$$
F_{a ; p}\left(z_{0}\right)=\frac{1}{2 \pi i} \int_{\mathcal{L}(r, R)} \frac{F_{a ; p}(\xi)}{\xi-z_{0}} \mathrm{~d} \xi,
$$

where $\mathcal{L}(r, R)$ is a positively oriented contour in $\mathbb{C} \backslash[0, \infty)$, as showed in Figure 1 , such that

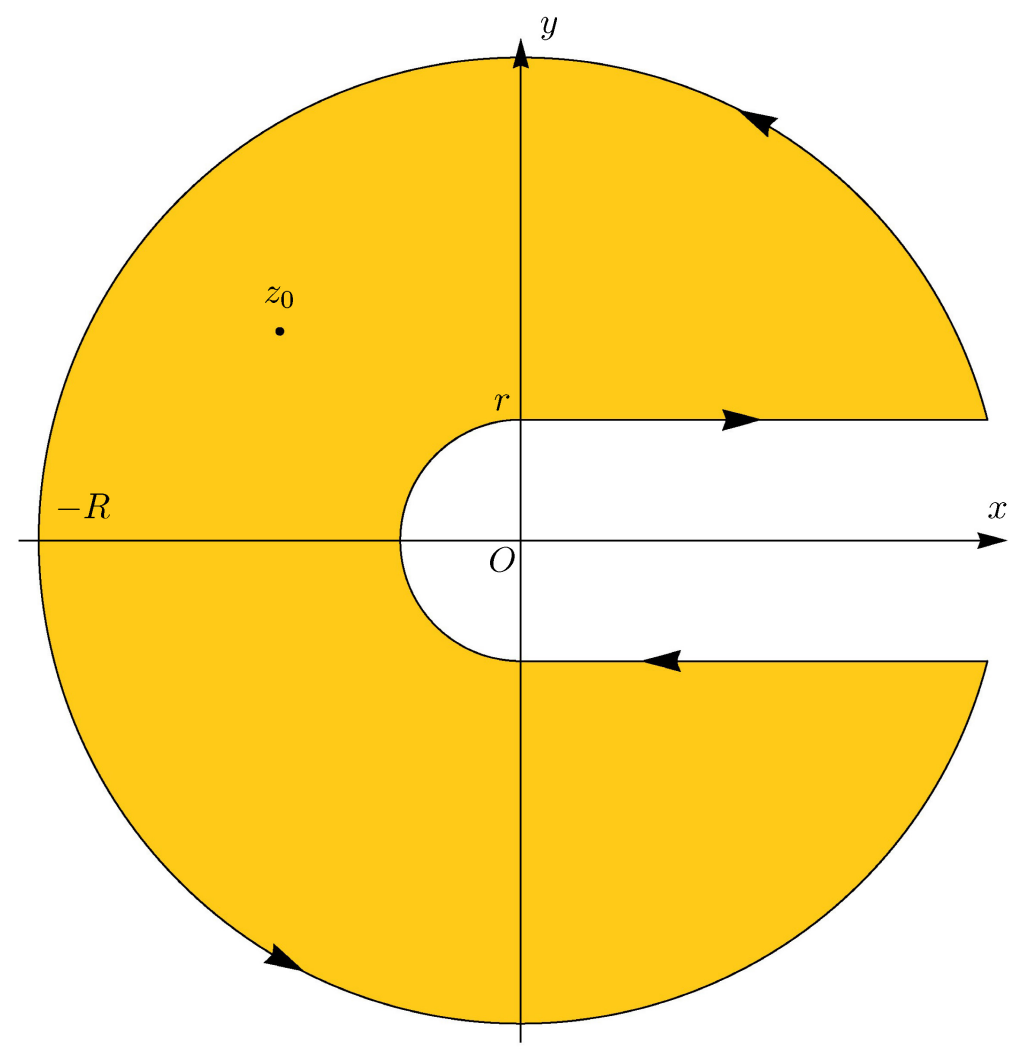

Figure 1 . The positively oriented contour $\mathcal{L}(r, R)$ in $\mathbb{C} \backslash[0, \infty)$

(1) $0<r<\left|z_{0}\right|<R$;

(2) $L(r, R)$ consists of the half circle $z=r e^{i \theta}$ for $\theta \in\left[\frac{\pi}{2}, \frac{3 \pi}{2}\right]$;

(3) $L(r, R)$ consists of the line segments $z=x \pm i r$ for $x \in(0, R(r)]$, where $R(r)=\sqrt{R^{2}-r^{2}}$; 
(4) $L(r, R)$ consists of the circular $\operatorname{arc} z=R e^{i \theta}$ for

$$
\theta \in\left(\arctan \frac{r}{R(r)}, 2 \pi-\arctan \frac{r}{R(r)}\right)=\left(\arcsin \frac{r}{R}, 2 \pi-\arcsin \frac{r}{R}\right) ;
$$

(5) the line segments $z=x \pm i r$ for $x \in(0, R(r)]$ cut the circle $|z|=R$ at the points $R(r) \pm i r$ and $R(r) \rightarrow R$ as $r \rightarrow 0^{+}$.

The integral on the circular $\operatorname{arc} z=R e^{i \theta}$ with positive orientation of the contour $\mathcal{L}(r, R)$ equals

$$
\begin{aligned}
& \frac{1}{2 \pi i} \int_{\arcsin (r / R)}^{2 \pi-\arcsin (r / R)} \frac{R i e^{i \theta}}{R e^{i \theta}-z_{0}} \frac{1}{a+\exp \left[p \ln \left(-R e^{i \theta}\right)\right]} \mathrm{d} \theta \\
= & \frac{1}{2 \pi} \int_{\arcsin (r / R)}^{2 \pi-\arcsin (r / R)} \frac{R e^{i \theta}}{R e^{i \theta}-z_{0}} \frac{1}{a+R^{p} \exp \left[i p \arg \left(-R e^{i \theta}\right)\right]} \mathrm{d} \theta
\end{aligned}
$$

which tends to 0 uniformly with respect to $\theta$ as $R \rightarrow \infty$, where we used the limits

$$
\lim _{R \rightarrow \infty} \frac{R e^{i \theta}}{R e^{i \theta}-z_{0}}=\lim _{R \rightarrow \infty} \frac{e^{i \theta}}{e^{i \theta}-z_{0} / R}=1
$$

and

$$
\lim _{R \rightarrow \infty}\left|R^{p} \exp \left[i p \arg \left(-R e^{i \theta}\right)\right]\right|=\lim _{R \rightarrow \infty} R^{p}=\infty .
$$

The integral on the half circle $z=r e^{i \theta}$ for $\theta \in\left[\frac{\pi}{2}, \frac{3 \pi}{2}\right]$ with positive orientation of the contour curve $\mathcal{L}(r, R)$ is

$$
\begin{aligned}
& \frac{1}{2 \pi i} \int_{3 \pi / 2}^{\pi / 2} \frac{r i e^{i \theta}}{r e^{i \theta}-z_{0}} \frac{1}{a+\exp \left[p \ln \left(-r e^{i \theta}\right)\right]} \mathrm{d} \theta \\
&=-\frac{1}{2 \pi} \int_{\pi / 2}^{3 \pi / 2} \frac{r e^{i \theta}}{r e^{i \theta}-z_{0}} \frac{1}{a+r^{p} \exp \left[i p \arg \left(-r e^{i \theta}\right)\right]} \mathrm{d} \theta \\
&=-\frac{1}{2 \pi} \int_{\pi / 2}^{3 \pi / 2} \frac{e^{i \theta}}{e^{i \theta}-z_{0} / r} \frac{1}{a+r^{p} \exp \left[i p \arg \left(-r e^{i \theta}\right)\right]} \mathrm{d} \theta
\end{aligned}
$$

which tends to 0 uniformly with respect to $\theta$ as $r \rightarrow 0^{+}$, where we used the limits

$$
\lim _{r \rightarrow 0^{+}} \frac{e^{i \theta}}{e^{i \theta}-z_{0} / r}=0 \quad \text { and } \quad \lim _{r \rightarrow 0^{+}}\left|r^{p} \exp \left[i p \arg \left(-r e^{i \theta}\right)\right]\right|=\lim _{r \rightarrow 0^{+}} r^{p}=0 .
$$

On the half line $z=x+i r$ for $x \in(0, R(r)]$ and $r>0$, a direct computation gives

$$
\begin{aligned}
F_{a ; p}(x+i r) & =\frac{1}{a+\exp [p \ln (-x-r i)]} \\
& =\frac{1}{a+\exp \left(p \ln \sqrt{x^{2}+r^{2}}+i p[\arctan (r / x)-\pi]\right)} \\
& \rightarrow \frac{1}{a+x^{p} \cos (p \pi)-i x^{p} \sin (p \pi)}, \quad r \rightarrow 0^{+} \\
& =\frac{a+x^{p} \cos (p \pi)+i x^{p} \sin (p \pi)}{\left[a+x^{p} \cos (p \pi)\right]^{2}+\left[x^{p} \sin (p \pi)\right]^{2}} \\
& =\frac{a+x^{p} \cos (p \pi)+i x^{p} \sin (p \pi)}{a^{2}+2 a x^{p} \cos (p \pi)+x^{2 p}} .
\end{aligned}
$$


Since $\overline{e^{z}}=e^{\bar{z}}$ and $\overline{\ln z}=\ln \bar{z}$, it follows that

$$
\begin{aligned}
\overline{F_{a ; p}(z)}= & \frac{\overline{1}}{\bar{a}+\overline{\exp [p \ln (-z)]}}=\frac{1}{a+\exp [\overline{p \ln (-z)}]}=\frac{1}{a+\exp [\bar{p} \overline{\ln (-z)}]} \\
& =\frac{1}{a+\exp [p \ln (\overline{-z})]}=\frac{1}{a+\exp [p \ln (-\bar{z})]}=F_{a ; p}(\bar{z}) .
\end{aligned}
$$

Accordingly, the integral on the line segments $z=x \pm i r$ for $x>0$ with positive orientation of the contour $\mathcal{L}(r, R)$ is equal to

$$
\begin{aligned}
& \frac{1}{2 \pi i}\left[\int_{0}^{R(r)} \frac{F_{a ; p}(x+i r)}{x+i r-z_{0}} \mathrm{~d} x+\int_{R(r)}^{0} \frac{F_{a ; p}(x-i r)}{x-i r-z_{0}} \mathrm{~d} x\right] \\
= & \frac{1}{2 \pi i} \int_{0}^{R(r)} \frac{\left(x-i r-z_{0}\right) F_{a ; p}(x+i r)-\left(x+i r-z_{0}\right) F_{a ; p}(x-i r)}{\left(x+i r-z_{0}\right)\left(x-i r-z_{0}\right)} \mathrm{d} x \\
= & \frac{1}{2 \pi i} \int_{0}^{R(r)} \frac{\left(x-z_{0}\right)\left[F_{a ; p}(x+i r)-F_{a ; p}(x-i r)\right]-i r\left[F_{a ; p}(x+i r)+F_{a ; p}(x-i r)\right]}{\left(x+i r-z_{0}\right)\left(x-i r-z_{0}\right)} \mathrm{d} x \\
= & \frac{1}{2 \pi i} \int_{0}^{R(r)} \frac{\left(x-z_{0}\right)\left[F_{a ; p}(x+i r)-F(\overline{x+i r})\right]-i r\left[F_{a ; p}(x+i r)+F(\overline{x+i r})\right]}{\left(x+i r-z_{0}\right)\left(x-i r-z_{0}\right)} \mathrm{d} x \\
= & \frac{1}{2 \pi i} \int_{0}^{R(r)} \frac{\left(x-z_{0}\right)\left[F_{a ; p}(x+i r)-\overline{F_{a ; p}(x+i r)}\right]-i r\left[F_{a ; p}(x+i r)+\overline{F_{a ; p}(x+i r)}\right]}{\left(x+i r-z_{0}\right)\left(x-i r-z_{0}\right)} \mathrm{d} x \\
= & \frac{1}{2 \pi i} \int_{0}^{R(r)} \frac{\left(x-z_{0}\right)\left[2 i \Im\left(F_{a ; p}(x+i r)\right)\right]-i r\left[2 \Re\left(F_{a ; p}(x+i r)\right)\right]}{\left(x+i r-z_{0}\right)\left(x-i r-z_{0}\right)} \mathrm{d} x \\
\rightarrow & \frac{1}{2 \pi i} \int_{0}^{\infty} \frac{2 i}{x-z_{0}} \frac{x^{p} \sin (p \pi)}{a^{2}+2 a x^{p} \cos (p \pi)+x^{2 p}} \mathrm{~d} x, \quad r \rightarrow 0^{+}, \quad R \rightarrow \infty \\
= & \frac{1}{\pi} \int_{0}^{\infty} \frac{1}{x-z_{0}} \frac{x^{p} \sin (p \pi)}{a^{2}+2 a x^{p} \cos (p \pi)+x^{2 p}} \mathrm{~d} x .
\end{aligned}
$$

Consequently, it follows that

$$
\frac{1}{a+\exp \left[p \ln \left(-z_{0}\right)\right]}=\frac{1}{\pi} \int_{0}^{\infty} \frac{1}{x-z_{0}} \frac{x^{p} \sin (p \pi)}{a^{2}+2 a x^{p} \cos (p \pi)+x^{2 p}} \mathrm{~d} x
$$

for $z_{0} \in \mathbb{C} \backslash[0, \infty)$ and $\arg z_{0} \in(0,2 \pi)$. Due to the point $z_{0}$ in $(3.2)$ being arbitrary, the integral formula 3.2 can be rewritten as

$$
F_{a ; p}(z)=\frac{1}{a+\exp [p \ln (-z)]}=\frac{\sin (p \pi)}{\pi} \int_{0}^{\infty} \frac{1}{t-z} \frac{t^{p}}{a^{2}+2 a \cos (p \pi) t^{p}+t^{2 p}} \mathrm{~d} t,
$$

where $z \in \mathbb{C} \backslash[0, \infty)$ and $\arg z \in(0,2 \pi)$.

For $a \geq 0, b>0,0<p<1$, and $\arg (z-b) \in(0,2 \pi)$, it is easy to see that $G_{a, b ; p}(z)=F_{a ; p}(z-b)$. Therefore, from $(3.3)$, it follows that

$$
G_{a, b ; p}(z)=\frac{\sin (p \pi)}{\pi} \int_{0}^{\infty} \frac{1}{b+t-z} \frac{t^{p}}{a^{2}+2 a \cos (p \pi) t^{p}+t^{2 p}} \mathrm{~d} t .
$$

The integral representation (3.1) is thus proved. The proof of Theorem 3.1 is complete.

Remark 3.1. When taking $z=x \in(-\infty, b)$, the integral representation (3.1) becomes

$$
\frac{1}{a+(b-x)^{p}}=\frac{\sin (p \pi)}{\pi} \int_{0}^{\infty} \frac{t^{p}}{a^{2}+2 a \cos (p \pi) t^{p}+t^{2 p}} \frac{1}{b+t-x} \mathrm{~d} t .
$$

When taking $x \rightarrow b^{-}$, the integral in 3.4 converges. Consequently, the integral representation $(3.4)$ is valid on $(-\infty, b]$. 
Remark 3.2. Taking $p=\frac{1}{2}$ in the integral representation (3.1) recovers the integral representation 1.5 .

Remark 3.3. When taking $p=\frac{1}{3}$ and $p=\frac{1}{4}$ respectively, the integral representation (3.1) becomes

$$
\frac{1}{a+\sqrt[3]{b-z}}=\frac{\sqrt{3}}{2 \pi} \int_{0}^{\infty} \frac{\sqrt[3]{t}}{a^{2}+a \sqrt[3]{t}+\sqrt[3]{t^{2}}} \frac{1}{b+t-z} \mathrm{~d} t
$$

and

$$
\frac{1}{a+\sqrt[4]{b-z}}=\frac{\sqrt{2}}{2 \pi} \int_{0}^{\infty} \frac{\sqrt[4]{t}}{a^{2}+\sqrt{2} a \sqrt[4]{t}+\sqrt{t}} \frac{1}{b+t-z} \mathrm{~d} t
$$

for $\arg (z-b) \in(0,2 \pi)$ respectively.

\section{An integral RePRESEntation For $\mathfrak{C}_{n}(a, b ; p)$}

From the integral representation (3.1) or (3.4), we can derive an integral representation for the functional sequence $\mathfrak{C}_{n}(a, b ; p)$ as follows.

Theorem 4.1. Let $a \geq 0, b>0$, and $0<p<1$ be real numbers. Then the functional sequence $\mathfrak{C}_{n}(a, b ; p)$ for $n \geq 0$ can be represented by

$$
\mathfrak{C}_{n}(a, b ; p)=\frac{\sin (p \pi)}{\pi} \int_{0}^{\infty} \frac{t^{p}}{a^{2}+2 a \cos (p \pi) t^{p}+t^{2 p}} \frac{1}{(b+t)^{n+1}} \mathrm{~d} t .
$$

Proof. Differentiating $n \geq 0$ times with respect to $z$ on both sides of 3.1 and taking the limit $z \rightarrow 0$ yield

$$
\begin{aligned}
\lim _{z \rightarrow 0}\left[G_{a, b ; p}(z)\right]^{(n)} & =\frac{\sin (p \pi)}{\pi} \lim _{z \rightarrow 0} \int_{0}^{\infty} \frac{t^{p}}{a^{2}+2 a \cos (p \pi) t^{p}+t^{2 p}} \frac{\mathrm{d}^{n}}{\mathrm{~d} z^{n}}\left(\frac{1}{b+t-z}\right) \mathrm{d} t \\
& =\frac{\sin (p \pi)}{\pi} \int_{0}^{\infty} \frac{t^{p}}{a^{2}+2 a \cos (p \pi) t^{p}+t^{2 p}} \lim _{z \rightarrow 0} \frac{n !}{(b+t-z)^{n+1}} \mathrm{~d} t \\
& =\frac{n ! \sin (p \pi)}{\pi} \int_{0}^{\infty} \frac{t^{p}}{a^{2}+2 a \cos (p \pi) t^{p}+t^{2 p}} \frac{1}{(b+t)^{n+1}} \mathrm{~d} t .
\end{aligned}
$$

As a result, by virtue of (1.7), we have

$$
\mathfrak{C}_{n}(a, b ; p)=\frac{1}{n !} \lim _{x \rightarrow 0} \frac{\mathrm{d}^{n} G_{a, b ; p}(x)}{\mathrm{d} x^{n}}=\frac{\sin (p \pi)}{\pi} \int_{0}^{\infty} \frac{t^{p}}{a^{2}+2 a \cos (p \pi) t^{p}+t^{2 p}} \frac{1}{(b+t)^{n+1}} \mathrm{~d} t .
$$

The integral representation (4.1) is thus proved. The proof of Theorem 4.1 is complete.

Remark 4.1. If taking $p=\frac{1}{2}$ in 4.1, one can recover 1.6 immediately.

Remark 4.2. When taking $p=\frac{1}{3}$ and $p=\frac{1}{4}$ respectively, the integral representation (4.1) becomes

$$
\mathfrak{C}_{n}\left(a, b ; \frac{1}{3}\right)=\frac{\sqrt{3}}{2 \pi} \int_{0}^{\infty} \frac{\sqrt[3]{t}}{a^{2}+a \sqrt[3]{t}+\sqrt[3]{t^{2}}} \frac{1}{(b+t)^{n+1}} \mathrm{~d} t
$$

and

$$
\mathfrak{C}_{n}\left(a, b ; \frac{1}{4}\right)=\frac{\sqrt{2}}{2 \pi} \int_{0}^{\infty} \frac{\sqrt[4]{t}}{a^{2}+\sqrt{2} a \sqrt[4]{t}+\sqrt{t}} \frac{1}{(b+t)^{n+1}} \mathrm{~d} t
$$

respectively. 


\section{TWO INTEGRAL FORMULAS}

Combining the explicit formula (2.1) in Theorem 2.1 with the integral representation (4.1) in Theorem 4.1 and combining (1.4) with (1.6), we can conclude two integral formulas.

Theorem 5.1. Let $a \geq 0$ and $b>0$ be real numbers and let $n \geq 0$ be integers. If $0<p<1$, then

$$
\begin{aligned}
\int_{0}^{\infty} \frac{t^{p}}{a^{2}+2 a \cos (p \pi) t^{p}+t^{2 p}} & \frac{1}{(b+t)^{n+1}} \mathrm{~d} t \\
= & \frac{\pi}{\left(a+b^{p}\right) \sin (p \pi)} \frac{(-1)^{n}}{n ! b^{n}} \sum_{k=0}^{n} \frac{1}{\left(1+a / b^{p}\right)^{k}} \sum_{\ell=0}^{k}(-1)^{\ell}\left(\begin{array}{c}
k \\
\ell
\end{array}\right)\langle p \ell\rangle_{n} .
\end{aligned}
$$

In particular, if $p=\frac{1}{2}$, then

$$
\int_{0}^{\infty} \frac{\sqrt{t}}{a+t} \frac{1}{(b+t)^{n+1}} \mathrm{~d} t=\frac{\pi}{(2 n) ! ! b^{n+1 / 2}} \sum_{k=0}^{n}\left(\begin{array}{c}
2 n-k-1 \\
2(n-k)
\end{array}\right) \frac{k ![2(n-k)-1] ! !}{(1+\sqrt{a / b})^{k+1}} .
$$

Alternative proof of the integral formula (5.1) for $a, b>0$ and $0<p<1$. The formula of Faà di Bruno and Schlömilch [14, p. 33] states that

$$
\frac{\mathrm{d}^{n}(f \circ g)}{\mathrm{d} x^{n}}=\sum_{k=1}^{n}\left(f^{(k)} \circ g\right) Y_{n, k}(g)
$$

where the operator $Y_{n, k}$ has the explicit form

$$
Y_{n, k}(g)=\frac{1}{k !} \sum_{j=0}^{k}(-1)^{k-j}\left(\begin{array}{l}
k \\
j
\end{array}\right) g^{k-j}\left(g^{j}\right)^{(n)}
$$

An inductive proof for this formula can also be found in 22 .

Consider the case $g(x)=x^{p}$ with $0<p<1$. According to (5.4), we have

$$
\begin{aligned}
Y_{n, k}\left(x^{p}\right) & =\frac{1}{k !} \sum_{\ell=0}^{k}(-1)^{k-\ell}\left(\begin{array}{l}
k \\
\ell
\end{array}\right) x^{(k-\ell) p}\left(x^{p \ell}\right)^{(n)} \\
& =\frac{1}{k !} \sum_{\ell=0}^{k}(-1)^{k-\ell}\left(\begin{array}{l}
k \\
\ell
\end{array}\right) x^{(k-\ell) p}\langle p \ell\rangle_{n} x^{p \ell-n} \\
& =\frac{x^{k p-n}}{k !} \sum_{\ell=0}^{k}(-1)^{k-\ell}\left(\begin{array}{l}
k \\
\ell
\end{array}\right)\langle p \ell\rangle_{n} .
\end{aligned}
$$

When setting $f(x)=\frac{1}{1+x}$, according to 5.3 , we have

$$
\begin{aligned}
\frac{\mathrm{d}^{n}}{\mathrm{~d} x^{n}}\left(\frac{1}{1+x^{p}}\right) & =\sum_{k=1}^{n} \frac{(-1)^{k} k !}{\left(1+x^{p}\right)^{k+1}} \frac{x^{k p-n}}{k !} \sum_{\ell=0}^{k}(-1)^{k-\ell}\left(\begin{array}{c}
k \\
\ell
\end{array}\right)\langle p \ell\rangle_{n} \\
& =\frac{1}{x^{n+p}} \sum_{k=1}^{n}\left(\frac{x^{p}}{1+x^{p}}\right)^{k+1} \sum_{\ell=0}^{k}(-1)^{\ell}\left(\begin{array}{c}
k \\
\ell
\end{array}\right)\langle p \ell\rangle_{n} .
\end{aligned}
$$

Let $0<p<1$ and $\lambda>0$. Consider the meromorphic function

$$
F(z)=\frac{1}{\left(1-\lambda e^{-z}\right)\left(1+e^{p z}\right)}
$$


Consider also the positively oriented rectangle $\mathcal{R}=\overline{A B C D}$ with vertices $A(-R-\pi i), B(R-\pi i)$, $C(R+\pi i)$, and $D(-R+\pi i)$ for $R>|\ln \lambda|$, as indicated in Figure 2 In the interior of the

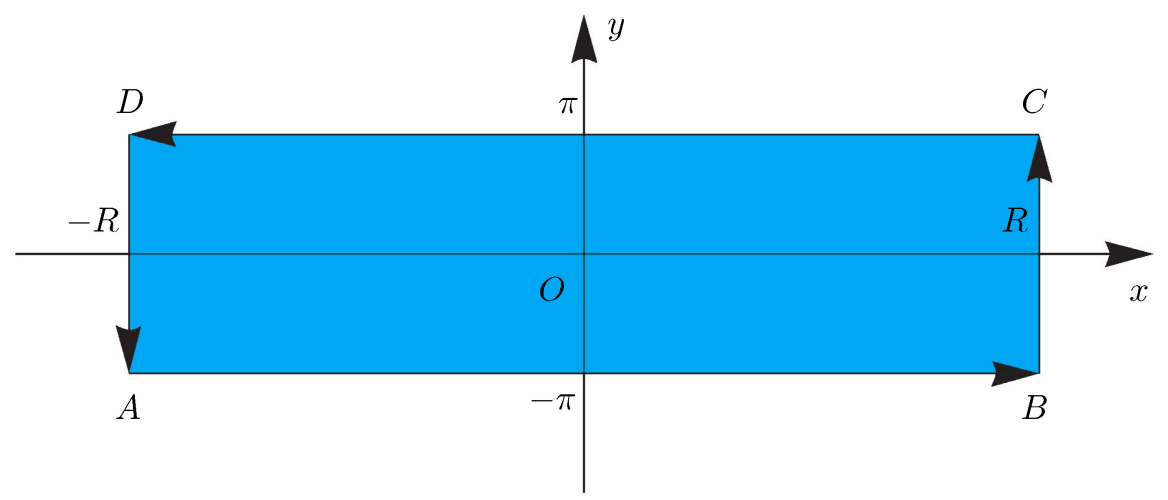

Figure 2. The positively oriented rectangle $\mathcal{R}=\overline{A B C D}$

region enclosed by the rectangle $\mathcal{R}$, the function $F$ has a unique pole $\mathfrak{p}=\ln \lambda$ of the residue $\operatorname{Res}(F, \mathfrak{p})=\frac{1}{1+\lambda^{p}}$. Therefore, by Cauchy's residue theorem in complex analysis, it follows that

$$
\int_{\mathcal{R}} F(z) \mathrm{d} z=\frac{2 \pi i}{1+\lambda^{p}} .
$$

On the other hand, from

$$
\left|\int_{\overline{B C}} F(z) \mathrm{d} z\right|=\left|i \int_{-\pi}^{\pi} F(R+i t) \mathrm{d} t\right| \leq \frac{\pi}{\left(1-\lambda e^{-R}\right)\left(e^{p R}-1\right)}
$$

and

we derive

$$
\left|\int_{\overline{D A}} F(z) \mathrm{d} z\right|=\left|i \int_{-\pi}^{\pi} F(-R+i t) \mathrm{d} t\right| \leq \frac{\pi}{\left(\lambda e^{R}-1\right)\left(1-e^{-p R}\right)},
$$

Further, we have

$$
\lim _{R \rightarrow \infty} \int_{\overline{B C}} F(z) \mathrm{d} z=\lim _{R \rightarrow \infty} \int_{\overline{D A}} F(z) \mathrm{d} z=0 .
$$

$$
\begin{aligned}
\int_{\overline{A B}} F(z) \mathrm{d} z+\int_{\overline{C D}} F(z) \mathrm{d} z & =\int_{-R}^{R} F(x-\pi i) \mathrm{d} x-\int_{-R}^{R} F(x+\pi i) \mathrm{d} x \\
& =\int_{-R}^{R} \frac{1}{1+\lambda e^{-x}}\left(\frac{1}{1+e^{p x-i p \pi}}-\frac{1}{1+e^{p x+i p \pi}}\right) \mathrm{d} x \\
& =2 i \sin (p \pi) \int_{-R}^{R} \frac{1}{1+\lambda e^{-x}} \frac{e^{p x}}{1+2 \cos (p \pi) e^{p x}+e^{2 p x}} \mathrm{~d} x .
\end{aligned}
$$

Consequently, letting $R$ tend to $\infty$ in (5.6) and rearranging lead to

$$
\int_{-\infty}^{\infty} \frac{1}{1+\lambda e^{-x}} \frac{e^{p x}}{1+2 \cos (p \pi) e^{p x}+e^{2 p x}} \mathrm{~d} x=\frac{\pi}{\sin (p \pi)} \frac{1}{1+\lambda^{p}} .
$$

The change of variables $e^{x}=t$ yields

$$
\int_{0}^{\infty} \frac{t^{p}}{1+2 \cos (p \pi) t^{p}+t^{2 p}} \frac{\mathrm{d} t}{t+\lambda}=\frac{\pi}{\sin (p \pi)} \frac{1}{1+\lambda^{p}} .
$$


Taking the $n$th derivative with respect to $\lambda$ arrives at

$$
\int_{0}^{\infty} \frac{t^{p}}{1+2 \cos (p \pi) t^{p}+t^{2 p}} \frac{\mathrm{d} t}{(t+\lambda)^{n+1}}=\frac{\pi}{\sin (p \pi)} \frac{(-1)^{n}}{n !} \frac{\mathrm{d}^{n}}{\mathrm{~d} \lambda^{n}}\left(\frac{1}{1+\lambda^{p}}\right) .
$$

Combining this with $(5.5)$ reveals

$$
\int_{0}^{\infty} \frac{t^{p}}{1+2 \cos (p \pi) t^{p}+t^{2 p}} \frac{\mathrm{d} t}{(t+\lambda)^{n+1}}=\frac{\pi}{\sin (p \pi)} \frac{(-1)^{n}}{n ! \lambda^{n+p}} \sum_{k=1}^{n}\left(\frac{\lambda^{p}}{1+\lambda^{p}}\right)^{k+1} \sum_{\ell=0}^{k}(-1)^{\ell}\left(\begin{array}{l}
k \\
\ell
\end{array}\right)\langle p \ell\rangle_{n} .
$$

Now the change of variables $t=a^{-1 / p} u$ with $a>0$ yields

$$
\begin{aligned}
& \int_{0}^{\infty} \frac{u^{p}}{a^{2}+2 a \cos (p \pi) u^{p}+u^{2 p}} \frac{a^{1+n / p} \mathrm{~d} u}{\left(u+a^{1 / p} \lambda\right)^{n+1}} \\
& \quad=\frac{\pi}{\sin (p \pi)} \frac{(-1)^{n}}{n ! \lambda^{n+p}} \sum_{k=1}^{n}\left(\frac{\lambda^{p}}{1+\lambda^{p}}\right)^{k+1} \sum_{\ell=0}^{k}(-1)^{\ell}\left(\begin{array}{l}
k \\
\ell
\end{array}\right)\langle p \ell\rangle_{n} .
\end{aligned}
$$

Finally, applying this to $\lambda=a^{-1 / p} b$ for $b>0$ results in

$$
\int_{0}^{\infty} \frac{u^{p}}{a^{2}+2 a \cos (p \pi) u^{p}+u^{2 p}} \frac{\mathrm{d} u}{(u+b)^{n+1}}=\frac{\pi}{\sin (p \pi)} \frac{(-1)^{n}}{n ! b^{n}} \sum_{k=1}^{n} \frac{b^{p k}}{\left(a+b^{p}\right)^{k+1}} \sum_{\ell=0}^{k}(-1)^{\ell}\left(\begin{array}{l}
k \\
\ell
\end{array}\right)\langle p \ell\rangle_{n}
$$

or, equivalently,

$$
\begin{aligned}
\int_{0}^{\infty} \frac{u^{p}}{a^{2}+2 a \cos (p \pi) u^{p}+u^{2 p}} \frac{\mathrm{d} u}{(u+b)^{n+1}} & \\
& =\frac{\pi}{\left(a+b^{p}\right) \sin (p \pi)} \frac{(-1)^{n}}{n ! b^{n}} \sum_{k=1}^{n} \frac{1}{\left(1+a / b^{p}\right)^{k}} \sum_{\ell=0}^{k}(-1)^{\ell}\left(\begin{array}{c}
k \\
\ell
\end{array}\right)\langle p \ell\rangle_{n} .
\end{aligned}
$$

The required proof is complete.

Remark 5.1. By analytic continuation, we claim that the integral formula (5.1) is valid for

$$
a \in\{z \in \mathbb{C}:|\arg (z)|<(1-p) \pi\}
$$

and $b \in \mathbb{C} \backslash(-\infty, 0]$. For example, if $0<p<\frac{1}{2}, b=1$, and $a=i$, then

$$
\int_{0}^{\infty} \frac{u^{p}}{-1+2 i \cos (p \pi) u^{p}+u^{2 p}} \frac{\mathrm{d} u}{(u+1)^{n+1}}=\frac{\pi}{\sin (p \pi)} \frac{(-1)^{n}}{n !} \sum_{k=0}^{n} \frac{1}{(1+i)^{k+1}} \sum_{\ell=0}^{k}(-1)^{\ell}\left(\begin{array}{l}
k \\
\ell
\end{array}\right)\langle p \ell\rangle_{n}
$$

which is equivalent to

$$
\begin{aligned}
\int_{0}^{\infty} \frac{u^{p}\left[u^{2 p}-1-2 i \cos (p \pi) u^{p}\right]}{1+2 \cos (2 p \pi) u^{2 p}+u^{4 p}} \frac{\mathrm{d} u}{(u+1)^{n+1}} & \\
& =\frac{\pi}{\sin (p \pi)} \frac{(-1)^{n}}{n !} \sum_{k=0}^{n} \frac{e^{-i \pi(k+1) / 4}}{2^{(k+1) / 2}} \sum_{\ell=0}^{k}(-1)^{\ell}\left(\begin{array}{c}
k \\
\ell
\end{array}\right)\langle p \ell\rangle_{n} .
\end{aligned}
$$

Further comparing imaginary parts of this equality gives

$$
\begin{aligned}
\int_{0}^{\infty} \frac{u^{2 p}}{1+2 \cos (2 p \pi) u^{2 p}+u^{4 p}} \frac{\mathrm{d} u}{(u+1)^{n+1}} & \\
& =\frac{\pi}{\sin (2 p \pi)} \frac{(-1)^{n}}{n !} \sum_{k=0}^{n} \frac{\sin (\pi(k+1) / 4)}{2^{(k+1) / 2}} \sum_{\ell=0}^{k}(-1)^{\ell}\left(\begin{array}{l}
k \\
\ell
\end{array}\right)\langle p \ell\rangle_{n} .
\end{aligned}
$$


On the other hand, setting $a=b=1$ and replacing $p$ by $2 p$ in (5.1) result in

$$
\int_{0}^{\infty} \frac{u^{2 p}}{1+2 \cos (2 p \pi) u^{p}+u^{2 p}} \frac{\mathrm{d} u}{(u+1)^{n+1}}=\frac{\pi}{\sin (2 p \pi)} \frac{(-1)^{n}}{n !} \sum_{k=0}^{n} \frac{1}{2^{k+1}} \sum_{\ell=0}^{k}(-1)^{\ell}\left(\begin{array}{l}
k \\
\ell
\end{array}\right)\langle 2 p \ell\rangle_{n} .
$$

Accordingly, for $0<p<\frac{1}{2}$, we obtain

$$
\sum_{k=0}^{n} \frac{1}{2^{k+1}} \sum_{\ell=0}^{k}(-1)^{\ell}\left(\begin{array}{c}
k \\
\ell
\end{array}\right)\langle 2 p \ell\rangle_{n}=\sum_{k=0}^{n} \frac{\sin (\pi(k+1) / 4)}{2^{(k+1) / 2}} \sum_{\ell=0}^{k}(-1)^{\ell}\left(\begin{array}{c}
k \\
\ell
\end{array}\right)\langle p \ell\rangle_{n} .
$$

Since both sides of this equality are polynomials in the variable $p$, we acquire

$$
\sum_{k=0}^{n} \frac{1}{2^{k+1}} \sum_{\ell=0}^{k}(-1)^{\ell}\left(\begin{array}{l}
k \\
\ell
\end{array}\right)\langle 2 \ell z\rangle_{n}=\sum_{k=0}^{n} \frac{\sin (\pi(k+1) / 4)}{2^{(k+1) / 2}} \sum_{\ell=0}^{k}(-1)^{\ell}\left(\begin{array}{l}
k \\
\ell
\end{array}\right)\langle\ell z\rangle_{n}
$$

for all $z \in \mathbb{C}$.

Remark 5.2. From the proof of Theorem 2.1, we conclude that

$$
\frac{\mathrm{d}^{n} G_{a, b ; p}(x)}{\mathrm{d} x^{n}}=\frac{(-1)^{n}}{(b-x)^{n}} \sum_{k=0}^{n} \frac{(b-x)^{k p}}{\left[a+(b-x)^{p}\right]^{k+1}} \sum_{\ell=0}^{k}(-1)^{\ell}\left(\begin{array}{c}
k \\
\ell
\end{array}\right)\langle p \ell\rangle_{n} .
$$

Combining this with the integral representation (3.1) in Theorem 3.1 derives the integral formula

$$
\begin{aligned}
& \int_{0}^{\infty} \frac{t^{p}}{a^{2}+2 a \cos (p \pi) t^{p}+t^{2 p}} \frac{1}{(b+t-x)^{n+1}} \mathrm{~d} t \\
& \quad=\frac{\pi}{\left[a+(b-x)^{p}\right] \sin (p \pi)} \frac{(-1)^{n}}{n !(b-x)^{n}} \sum_{k=0}^{n} \frac{1}{\left[1+a /(b-x)^{p}\right]^{k}} \sum_{\ell=0}^{k}(-1)^{\ell}\left(\begin{array}{c}
k \\
\ell
\end{array}\right)\langle p \ell\rangle_{n} .
\end{aligned}
$$

This is essentially same as the integral formula 5.1 .

Remark 5.3. Letting $a=0$ and computing integrals in 5.1 and (5.2) leads to

$$
\int_{0}^{\infty} \frac{1}{t^{p}} \frac{1}{(b+t)^{n+1}} \mathrm{~d} t=\frac{\Gamma(1-p) \Gamma(n+p)}{n ! b^{n+p}}=\frac{\pi}{b^{p} \sin (p \pi)} \frac{(-1)^{n}}{n ! b^{n}} \sum_{k=0}^{n} \sum_{\ell=0}^{k}(-1)^{\ell}\left(\begin{array}{l}
k \\
\ell
\end{array}\right)\langle p \ell\rangle_{n}
$$

and

$$
\int_{0}^{\infty} \frac{1}{\sqrt{t}(b+t)^{n+1}} \mathrm{~d} t=\frac{\sqrt{\pi} \Gamma\left(n+\frac{1}{2}\right)}{n ! b^{n+1 / 2}}=\frac{\pi}{(2 n) ! ! b^{n+1 / 2}} \sum_{k=0}^{n} k ![2(n-k)-1] ! !\left(\begin{array}{c}
2 n-k-1 \\
2(n-k)
\end{array}\right) .
$$

As a result, we obtain two interesting formulas

$$
\Gamma(1-p) \Gamma(n+p)=\frac{\pi}{\sin (p \pi)}(-1)^{n} \sum_{k=0}^{n} \sum_{\ell=0}^{k}(-1)^{\ell}\left(\begin{array}{l}
k \\
\ell
\end{array}\right)\langle p \ell\rangle_{n}
$$

and

$$
\Gamma\left(n+\frac{1}{2}\right)=\frac{\sqrt{\pi}}{2^{n}} \sum_{k=0}^{n} k ![2(n-k)-1] ! !\left(\begin{array}{c}
2 n-k-1 \\
2(n-k)
\end{array}\right)
$$

for $0<p<1$ and $n \geq 0$. 
By the recurrent relation $\Gamma(z+1)=z \Gamma(z)$ and the reflection relation $\Gamma(z) \Gamma(1-z)=\frac{\pi}{\sin (z \pi)}$ for $z \neq 0, \pm 1, \ldots$ (see [25, Chapter 5]), we can rewrite the identity (5.7) as

$$
\begin{aligned}
(p)_{n} & =(-1)^{n} \sum_{k=0}^{n} \sum_{\ell=0}^{k}(-1)^{\ell}\left(\begin{array}{c}
k \\
\ell
\end{array}\right)\langle p \ell\rangle_{n} \\
& =(-1)^{n} \sum_{\ell=0}^{n}(-1)^{\ell}\langle p \ell\rangle_{n} \sum_{k=\ell}^{n}\left(\begin{array}{c}
k \\
\ell
\end{array}\right) \\
& =(-1)^{n} \sum_{\ell=0}^{n}(-1)^{\ell}\langle p \ell\rangle_{n} \sum_{k=\ell}^{n}\left[\left(\begin{array}{c}
k+1 \\
\ell+1
\end{array}\right)-\left(\begin{array}{c}
k \\
\ell+1
\end{array}\right)\right] \\
& =(-1)^{n} \sum_{\ell=0}^{n}(-1)^{\ell}\left(\begin{array}{c}
n+1 \\
\ell+1
\end{array}\right)\langle p \ell\rangle_{n}
\end{aligned}
$$

for $0<p<1$ and $n \geq 0$, where the notation

$$
(\alpha)_{n}=\prod_{k=0}^{n-1}(\alpha+k)= \begin{cases}\alpha(\alpha+1) \cdots(\alpha+n-1), & n \geq 1 \\ 1, & n=0\end{cases}
$$

for $\alpha \in \mathbb{C}$ is called the rising factorial, the Pochhammer symbol, or shifted factorial. Because all sides in (5.9) are polynomials in $p$ for $0<p<1$, all equalities in (5.9) must be valid for all $p \in \mathbb{C}$ and $n \geq 0$. Thus, we obtain the identity

$$
(z)_{n}=(-1)^{n} \sum_{\ell=0}^{n}(-1)^{\ell}\left(\begin{array}{l}
n+1 \\
\ell+1
\end{array}\right)\langle\ell z\rangle_{n}, \quad z \in \mathbb{C} .
$$

By the recurrent relation $\Gamma(z+1)=z \Gamma(z)$ and the formula $\Gamma\left(\frac{1}{2}\right)=\sqrt{\pi}$ (see [25, Chapter 5]), we can rewrite the identity $(5.8)$ as

$$
(2 n-1) ! !=\sum_{k=0}^{n} k ![2(n-k)-1] ! !\left(\begin{array}{c}
2 n-k-1 \\
2(n-k)
\end{array}\right), \quad n \geq 0 .
$$

By the way, we can rearrange [12, Theorem 2.1] as

$$
\frac{1}{(2 n-1) ! !}=\frac{(-1)^{n+1}}{2^{3 n-2}(n-1) !} \sum_{k=0}^{2 n-1} \frac{(-1)^{k}}{2 n-2 k-1}\left(\begin{array}{c}
2 n-1 \\
k
\end{array}\right), \quad n \geq 1 .
$$

6. An elementary computation of the integral in 5.2

In this section, we will compute the integral in 5.2 elementarily.

Lemma 6.1. For real numbers $a, b$ with $a \neq b$ and any positive integer $n$, we have

$$
\frac{1}{(a+x)(b+x)^{n}}=\frac{1}{(b-a)^{n}(a+x)}-\sum_{\ell=1}^{n} \frac{1}{(b-a)^{n-\ell+1}(b+x)^{\ell}} .
$$

Proof. The equality (6.1) can be proved straightforwardly by induction on $n$. However, we will demonstrate an alternative proof as follow.

The right hand side of the equality (6.1) can be restructured and computed as

$$
\begin{aligned}
\frac{1}{(b-a)^{n+1}}\left[\frac{b-a}{a+x}-\sum_{\ell=1}^{n}\left(\frac{b-a}{b+x}\right)^{\ell}\right] & =\frac{1}{(b-a)^{n+1}}\left[\frac{b-a}{a+x}-\frac{b-a}{a+x}\left(1-\left(\frac{b-a}{b+x}\right)^{n}\right)\right] \\
& \left.=\frac{1}{(b-a)^{n+1}}\left[\frac{b-a}{a+x}\left(\frac{b-a}{b+x}\right)^{n}\right)\right]
\end{aligned}
$$


The proof of Lemma 6.1 is complete.

$$
=\frac{1}{(a+x)(b+x)^{n}} .
$$

Lemma 6.2. For real number $b>0$ and integer $\ell \geq 1$, we have

$$
\int_{0}^{\infty} \frac{\sqrt{t}}{(b+t)^{\ell+1}} \mathrm{~d} t=\frac{\pi}{b^{\ell-1 / 2}} \frac{(2 \ell-3) ! !}{(2 \ell) ! !} .
$$

For $a, b \geq 0$, we have

$$
\int_{0}^{\infty} \sqrt{t}\left(\frac{1}{a+t}-\frac{1}{b+t}\right) \mathrm{d} t=\pi(\sqrt{b}-\sqrt{a}) .
$$

Proof. It is straightforward that

$$
\begin{gathered}
\int_{0}^{\infty} \frac{\sqrt{t}}{(b+t)^{\ell+1}} \mathrm{~d} t=\frac{1}{b^{\ell+1}} \int_{0}^{\infty} \frac{\sqrt{t}}{(1+t / b)^{\ell+1}} \mathrm{~d} t=\frac{1}{b^{\ell-1 / 2}} \int_{0}^{\infty} \frac{\sqrt{u}}{(1+u)^{\ell+1}} \mathrm{~d} u \\
=\frac{1}{b^{\ell-1 / 2}} B\left(\frac{3}{2}, \ell-\frac{1}{2}\right)=\frac{1}{b^{\ell-1 / 2}} \frac{\Gamma\left(\frac{3}{2}\right) \Gamma\left(\ell-\frac{1}{2}\right)}{\Gamma(\ell+1)} \\
=\frac{1}{b^{\ell-1 / 2}} \frac{\frac{1}{2} \Gamma\left(\frac{1}{2}\right)\left(\ell-\frac{3}{2}\right)\left(\ell-\frac{5}{2}\right) \cdots \frac{3}{2} \frac{1}{2} \Gamma\left(\frac{1}{2}\right)}{\ell !}=\frac{\pi}{b^{\ell-1 / 2}} \frac{(2 \ell-3) ! !}{(2 \ell) ! !},
\end{gathered}
$$

where $B(z, w)$ denotes the classical beta function

$$
B(z, w)=\int_{0}^{1} t^{z-1}(1-t)^{w-1} \mathrm{~d} t=\int_{0}^{\infty} \frac{t^{z-1}}{(1+t)^{z+w}} \mathrm{~d} t
$$

for $\Re(z)>0$ and $\Re(w)>0$ and satisfies

$$
B(z, w)=\frac{\Gamma(z) \Gamma(w)}{\Gamma(z+w)}=B(w, z) .
$$

See [1, p. 258, 6.2.1 and 6.2.2].

When $a, b>0$ and $a \neq b$, the first proof of the formula 6.3 is straightforward as follows:

$$
\begin{aligned}
\int_{0}^{\infty} \sqrt{t}\left(\frac{1}{a+t}-\frac{1}{b+t}\right) \mathrm{d} t & =2 \int_{0}^{\infty} s^{2}\left(\frac{1}{a+s^{2}}-\frac{1}{b+s^{2}}\right) \mathrm{d} s \\
& =2(b-a) \int_{0}^{\infty} \frac{s^{2}}{\left(a+s^{2}\right)\left(b+s^{2}\right)} \mathrm{d} s \\
& =2(b-a) \int_{0}^{\infty} \frac{\mathrm{d}}{\mathrm{d} s}\left[\frac{\sqrt{a} \arctan \frac{s}{\sqrt{a}}-\sqrt{b} \arctan \frac{s}{\sqrt{b}}}{a-b}\right] \mathrm{d} s \\
& =\left.2(b-a) \frac{\sqrt{a} \arctan \frac{s}{\sqrt{a}}-\sqrt{b} \arctan \frac{s}{\sqrt{b}}}{a-b}\right|_{s=0} ^{s=\infty} \\
& =\pi(\sqrt{b}-\sqrt{a}) .
\end{aligned}
$$

When $a, b>0$ and $a \neq b$, we can alternatively prove the formula 6.3 as follows:

$$
\begin{aligned}
\int_{0}^{\infty} \sqrt{t}\left(\frac{1}{a+t}-\frac{1}{b+t}\right) \mathrm{d} t & =\int_{0}^{\infty}\left[\frac{b}{(b+t) \sqrt{t}}-\frac{a}{(a+t) \sqrt{t}}\right] \mathrm{d} t \\
& =\left.2 \sqrt{b} \arctan \sqrt{\frac{t}{b}}\right|_{t=0} ^{t=\infty}-\left.2 \sqrt{a} \arctan \sqrt{\frac{t}{a}}\right|_{t=0} ^{t=\infty} \\
& =\pi(\sqrt{b}-\sqrt{a}) .
\end{aligned}
$$


The proof of Lemma 6.2 is complete.

Theorem 6.1. If $n \geq 0$ is an integer and $a \geq 0, b>0$, and $a \neq b$ are real umbers, then

$$
\int_{0}^{\infty} \frac{\sqrt{t}}{a+t} \frac{1}{(b+t)^{n+1}} \mathrm{~d} t=-\frac{\pi}{(b-a)^{n+1}}\left[\sqrt{a}+\sqrt{b} \sum_{\ell=0}^{n}\left(1-\frac{a}{b}\right)^{\ell} \frac{(2 \ell-3) ! !}{(2 \ell) ! !}\right]
$$

where $(-3) ! !=-1$ and $(-1) ! !=0 ! !=1$.

First proof of Theorem 6.1. From Lemma 6.1, it follows that

$$
\frac{1}{(a+t)(b+t)^{n+1}}=\frac{1}{(b-a)^{n+1}}\left(\frac{1}{a+t}-\frac{1}{b+t}\right)-\sum_{\ell=2}^{n+1} \frac{1}{(b-a)^{n-\ell+2}(b+t)^{\ell}} .
$$

Further integrating on both sides with respect to $t$ and applying the formulas $\sqrt{6.2}$ and $(6.3)$ in Lemma 6.2 arrive at

$$
\begin{aligned}
\int_{0}^{\infty} \frac{\sqrt{t}}{a+t} \frac{1}{(b+t)^{n+1}} \mathrm{~d} t= & \frac{1}{(b-a)^{n+1}} \int_{0}^{\infty} \sqrt{t}\left(\frac{1}{a+t}-\frac{1}{b+t}\right) \mathrm{d} t \\
& -\int_{0}^{\infty} \sum_{\ell=2}^{n+1} \frac{\sqrt{t}}{(b-a)^{n-\ell+2}(b+t)^{\ell}} \mathrm{d} t \\
= & \frac{\pi(\sqrt{b}-\sqrt{a})}{(b-a)^{n+1}}-\sum_{\ell=1}^{n} \frac{1}{(b-a)^{n-\ell+1}} \int_{0}^{\infty} \frac{\sqrt{t}}{(b+t)^{\ell+1}} \mathrm{~d} t \\
= & \frac{\pi(\sqrt{b}-\sqrt{a})}{(b-a)^{n+1}}-\sum_{\ell=1}^{n} \frac{1}{(b-a)^{n-\ell+1}} \frac{\pi}{b^{\ell-1 / 2}} \frac{(2 \ell-3) ! !}{(2 \ell) ! !} \\
= & \frac{\pi(\sqrt{b}-\sqrt{a})}{(b-a)^{n+1}}-\frac{\pi \sqrt{b}}{(b-a)^{n+1}} \sum_{\ell=1}^{n}\left(1-\frac{a}{b}\right)^{\ell} \frac{(2 \ell-3) ! !}{(2 \ell) ! !} \\
= & -\frac{\pi}{(b-a)^{n+1}}\left[\sqrt{a}+\sqrt{b} \sum_{\ell=0}^{n}\left(1-\frac{a}{b}\right)^{\ell} \frac{(2 \ell-3) ! !}{(2 \ell) ! !}\right]
\end{aligned}
$$

The proof of Theorem 6.1 is complete.

Second proof of Theorem 6.1 . The formula 6.3 can be rewritten as

$$
\int_{0}^{\infty} \frac{\sqrt{t}}{(a+t)(b+t)} \mathrm{d} t=\pi\left(\frac{\sqrt{b}}{b-a}-\frac{\sqrt{a}}{b-a}\right) .
$$

Differentiating $n$ times with respect to $b$ on both sides of the above equality and making use of Leibnitz's rule for differentiation yield

$$
\begin{gathered}
\int_{0}^{\infty} \frac{(-1)^{n} n ! \sqrt{t}}{(a+t)(b+t)^{n+1}} \mathrm{~d} t=\pi\left[\frac{\mathrm{d}^{n}}{\mathrm{~d} b^{n}}\left(\frac{\sqrt{b}}{b-a}\right)-\frac{\mathrm{d}^{n}}{\mathrm{~d} b^{n}}\left(\frac{\sqrt{a}}{b-a}\right)\right] \\
=\pi\left[\sum_{\ell=0}^{n}\left(\begin{array}{l}
n \\
\ell
\end{array}\right) \frac{\mathrm{d}^{\ell}}{\mathrm{d} b^{\ell}}(\sqrt{b}) \frac{\mathrm{d}^{n-\ell}}{\mathrm{d} b^{n-\ell}}\left(\frac{1}{b-a}\right)-\frac{\sqrt{a}(-1)^{n} n !}{(b-a)^{n+1}}\right] \\
=\pi\left[\sum_{\ell=0}^{n}\left(\begin{array}{l}
n \\
\ell
\end{array}\right)\left\langle\frac{1}{2}\right\rangle_{\ell} b^{1 / 2-\ell} \frac{(-1)^{n-\ell}(n-\ell) !}{(b-a)^{n-\ell+1}}-\frac{\sqrt{a}(-1)^{n} n !}{(b-a)^{n+1}}\right] \\
=-\pi \frac{(-1)^{n} n !}{(b-a)^{n+1}}\left[\sqrt{a}-\frac{(b-a)^{n+1}}{(-1)^{n} n !} \sum_{\ell=0}^{n}\left(\begin{array}{c}
n \\
\ell
\end{array}\right)\left\langle\frac{1}{2}\right\rangle_{\ell} b^{1 / 2-\ell} \frac{(-1)^{n-\ell}(n-\ell) !}{(b-a)^{n-\ell+1}}\right]
\end{gathered}
$$




$$
\begin{gathered}
=-\pi \frac{(-1)^{n} n !}{(b-a)^{n+1}}\left[\sqrt{a}-\sqrt{b} \sum_{\ell=0}^{n} \frac{(-1)^{\ell}}{\ell !}\left\langle\frac{1}{2}\right\rangle_{\ell}\left(1-\frac{a}{b}\right)^{\ell}\right] \\
=-\pi \frac{(-1)^{n} n !}{(b-a)^{n+1}}\left[\sqrt{a}-\sqrt{b} \sum_{\ell=0}^{n} \frac{(-1)^{\ell}}{\ell !} \frac{1}{2}\left(-\frac{1}{2}\right) \cdots\left(\frac{1}{2}-\ell+1\right)\left(1-\frac{a}{b}\right)^{\ell}\right] \\
=-\pi \frac{(-1)^{n} n !}{(b-a)^{n+1}}\left[\sqrt{a}+\sqrt{b} \sum_{\ell=0}^{n} \frac{(2 \ell-3) ! !}{(2 \ell) ! !}\left(1-\frac{a}{b}\right)^{\ell}\right] .
\end{gathered}
$$

The integral formula 6.4 is thus proved.

Remark 6.1. The formula (6.3) can also be rearranged as

$$
\int_{0}^{\infty} \frac{\sqrt{t}}{(a+t)(b+t)} \mathrm{d} t=\frac{\pi}{\sqrt{a}+\sqrt{b}} .
$$

Differentiating $n$ times with respect to $b$ on both sides of the above equality, utilizing Faà di Bruno's formula (2.2), making use of the identity (2.3), and employing the formula (2.6) reveal

$$
\begin{gathered}
\int_{0}^{\infty} \frac{(-1)^{n} n ! \sqrt{t}}{(a+t)(b+t)^{n+1}} \mathrm{~d} t=\frac{\mathrm{d}^{n}}{\mathrm{~d} b^{n}}\left(\frac{\pi}{\sqrt{a}+\sqrt{b}}\right) \\
=\left.\sum_{k=0}^{n} \frac{\mathrm{d}^{k}}{\mathrm{~d} x^{k}}\left(\frac{\pi}{\sqrt{a}+x}\right)\right|_{x=\sqrt{b}} \mathrm{~B}_{n, k}\left((\sqrt{b})^{\prime},(\sqrt{b})^{\prime \prime}, \ldots,(\sqrt{b})^{n-k+1}\right) \\
=\left.\pi \sum_{k=0}^{n} \frac{(-1)^{k} k !}{(\sqrt{a}+x)^{k+1}}\right|_{x=\sqrt{b}} \mathrm{~B}_{n, k}\left(b^{1 / 2-1}\left\langle\frac{1}{2}\right\rangle_{1}, b^{1 / 2-2}\left\langle\frac{1}{2}\right\rangle_{2}, \ldots, b^{1 / 2-(n-k+1)}\left\langle\frac{1}{2}\right\rangle_{n-k+1}\right) \\
=\pi \sum_{k=0}^{n} \frac{(-1)^{k} k !}{(\sqrt{a}+\sqrt{b})^{k+1}} b^{k / 2-n} \mathrm{~B}_{n, k}\left(\left\langle\frac{1}{2}\right\rangle_{1},\left\langle\frac{1}{2}\right\rangle_{2}, \ldots,\left\langle\frac{1}{2}\right\rangle_{n-k+1}\right) \\
=\pi \sum_{k=0}^{n} \frac{(-1)^{k} k !}{(\sqrt{a}+\sqrt{b})^{k+1}} b^{k / 2-n}(-1)^{n+k}[2(n-k)-1] ! !\left(\frac{1}{2}\right)^{n}\left(\begin{array}{c}
2 n-k-1 \\
2(n-k)
\end{array}\right)
\end{gathered}
$$

which recovers the integral formula 5.2 , where $a \geq 0$ and $b>0$.

Remark 6.2. For $a \geq 0$ and $b>0$, combining 5.2 and 6.4 results in an identity

$$
\sum_{k=0}^{n}\left(\begin{array}{c}
2 n-k-1 \\
2(n-k)
\end{array}\right) \frac{k ![2(n-k)-1] ! !}{(1+\sqrt{a / b})^{k+1}}=-\frac{(2 n) ! !}{(1-a / b)^{n+1}}\left[\sqrt{\frac{a}{b}}+\sum_{\ell=0}^{n}\left(1-\frac{a}{b}\right)^{\ell} \frac{(2 \ell-3) ! !}{(2 \ell) ! !}\right]
$$

which can be further simplified as

$$
\sum_{k=0}^{n}\left(\begin{array}{c}
2 n-k-1 \\
2(n-k)
\end{array}\right) \frac{k ![2(n-k)-1] ! !}{(1+x)^{k+1}}=-\frac{(2 n) ! !}{\left(1-x^{2}\right)^{n+1}}\left[x+\sum_{\ell=0}^{n} \frac{(2 \ell-3) ! !}{(2 \ell) ! !}\left(1-x^{2}\right)^{\ell}\right]
$$

for $x \geq 0$ with $(-3) ! !=-1$ and $(-1) ! !=0 ! !=1$.

More extensively, the identity $(6.5)$ is valid for all $x \in \mathbb{C} \backslash\{-1,1\}$, because both sides of 6.5 are analytic on the set $\mathbb{C} \backslash\{-1,1\}$.

Remark 6.3. We note that all proofs in this section are elementary. In other words, Cauchy's integral formula in complex analysis is not employed in all proofs of Lemma 6.1. Lemma6.2, and Theorem 6.1 


\section{More REMARKS}

Finally, we list more remarks on main results of this paper.

Remark 7.1. Let $a \geq 0, b>0$, and $0<p<1$. Then, motivated by (4.1), one can consider the function

$$
\mathfrak{C}(a, b ; p ; x)=\frac{\sin (p \pi)}{\pi} \int_{0}^{\infty} \frac{t^{p}}{a^{2}+2 a \cos (p \pi) t^{p}+t^{2 p}} \frac{1}{(b+t)^{x+1}} \mathrm{~d} t
$$

for $x \geq 0$. Can one compute this integral explicitly in terms of closed forms? For more details on so-called closed forms, please refer to 4 and closely related references therein.

Remark 7.2. We do not find integral formulas (5.1), (5.2), and 6.4 in monographs and handbooks [1, 9, 25, 56.

Remark 7.3. Integral representations of the Catalan numbers $C_{n}$ for $n \geq 0$ have been reviewed and surveyed in [34, Section 2]. The first integral representation

$$
C_{n}=\frac{1}{2 \pi} \int_{0}^{4} \sqrt{\frac{4-x}{x}} x^{n} \mathrm{~d} x, \quad n \geq 0
$$

was discovered in [26] and applied in [49]. An alternative integral representation

$$
C_{n}=\frac{1}{\pi} \int_{0}^{\infty} \frac{\sqrt{t}}{(t+1 / 4)^{n+2}} \mathrm{~d} t
$$

was derived in [44, Theorem 1.3]. The equivalence of integral representations (7.1) and (7.2) was proved in [30, Theorem 1.3]. The integral representation (7.2) can be recovered from (4.1) by setting $a=\frac{1}{2}, b=\frac{1}{4}$, and $p=\frac{1}{2}$.

Remark 7.4. For $\alpha \in \mathbb{R}$, we consider the sequence $Z_{n, k}(\alpha)$ generated by

$$
\frac{\left[(1+T)^{\alpha}-1\right]^{k}}{k !}=\sum_{n \geq k \geq 0} Z_{n, k}(\alpha) \frac{T^{n}}{n !} .
$$

See [6, p. 158]. When $\alpha=\frac{1}{2}$, straightforward computation yields

which means that

$$
\begin{aligned}
\frac{\left[(1+T)^{1 / 2}-1\right]^{k}}{k !} & =\frac{1}{k !} \sum_{\ell=0}^{k}(-1)^{k-\ell}\left(\begin{array}{c}
k \\
\ell
\end{array}\right)(1+T)^{\ell / 2} \\
& =\frac{1}{k !} \sum_{\ell=0}^{k}(-1)^{k-\ell}\left(\begin{array}{c}
k \\
\ell
\end{array}\right) \sum_{n=0}^{\infty}\left(\begin{array}{c}
\ell / 2 \\
n
\end{array}\right) T^{n} \\
& =\sum_{n=0}^{\infty}\left[\frac{1}{k !} \sum_{\ell=0}^{k}(-1)^{k-\ell}\left(\begin{array}{c}
k \\
\ell
\end{array}\right)\left\langle\frac{\ell}{2}\right\rangle_{n}\right] \frac{T^{n}}{n !}
\end{aligned}
$$

$$
Z_{n, k}\left(\frac{1}{2}\right)=\frac{(-1)^{k}}{k !} \sum_{\ell=0}^{n}(-1)^{\ell}\left(\begin{array}{l}
k \\
\ell
\end{array}\right)\left\langle\frac{\ell}{2}\right\rangle_{n} .
$$

On the other hand, it is listed in [6, p. 158] that

Accordingly, we obtain the equality

$$
Z_{n, k}\left(\frac{1}{2}\right)=(-1)^{n-k} \frac{(n-1) !}{(k-1) !}\left(\begin{array}{c}
2 n-k-2 \\
n-1
\end{array}\right) \frac{1}{2^{2 n-k}} .
$$

$$
\frac{(-1)^{k}}{k !} \sum_{\ell=0}^{n}(-1)^{\ell}\left(\begin{array}{l}
k \\
\ell
\end{array}\right)\left\langle\frac{\ell}{2}\right\rangle_{n}=(-1)^{n-k} \frac{(n-1) !}{(k-1) !}\left(\begin{array}{c}
2 n-k-2 \\
n-1
\end{array}\right) \frac{1}{2^{2 n-k}}
$$


which can be rearranged as (2.5). Consequently, we recover the identity (2.5).

Remark 7.5. This paper is an expanded version of the electronic preprint [19].

\section{REFERENCES}

[1] M. Abramowitz and I. A. Stegun (Eds), Handbook of Mathematical Functions with Formulas, Graphs, and Mathematical Tables, National Bureau of Standards, Applied Mathematics Series 55, 10th printing, Dover Publications, New York and Washington, 1972.

[2] J.-C. Aval, Multivariate Fuss-Catalan numbers, Discrete Math. 308 (2008), no. 20, 4660-4669; available online at https://doi.org/10.1016/j.disc.2007.08.100

[3] C. Ballot, Lucasnomial Fuss-Catalan numbers and related divisibility questions, J. Integer Seq. 21 (2018), no. 6, Art. 18.6.5, 36 pages.

[4] J. M. Borwein and R. E. Crandall, Closed forms: what they are and why we care, Notices Amer. Math. Soc. 60 (2013), no. 1, 50-65; available online at https://doi.org/10.1090/noti936.

[5] W.-C. Chu, A new combinatorial interpretation for generalized Catalan number, Discrete Math. 65 (1987), no. 1, 91-94; available online at https://doi.org/10.1016/0012-365X(87)90214-7

[6] L. Comtet, Advanced Combinatorics: The Art of Finite and Infinite Expansions, Revised and Enlarged Edition, D. Reidel Publishing Co., Dordrecht and Boston, 1974; available online at https://doi.org/10. 1007/978-94-010-2196-8

[7] S. J. Dilworth and S. R. Mane, Applications of Fuss-Catalan numbers to success runs of Bernoulli trials, J. Probab. Stat. 2016, Art. ID 2071582, 13 pages; available online at https://doi.org/10.1155/2016/2071582

[8] N. I. Fuss, Solutio quaestionis, quot modis polygonum $n$ laterum in polygona $m$ laterum, per diagonales resolvi queat, Nova Acta Academiae Sci. Petropolitanae 9 (1791), 243-251.

[9] I. S. Gradshteyn and I. M. Ryzhik, Table of Integrals, Series, and Products, Translated from the Russian, Translation edited and with a preface by Daniel Zwillinger and Victor Moll, Eighth edition, Revised from the seventh edition, Elsevier/Academic Press, Amsterdam, 2015; available online at https://doi.org/10. 1016/B978-0-12-384933-5.00013-8

[10] R. L. Graham, D. E. Knuth, and O. Patashnik, Concrete Mathematics - A Foundation for Computer Science, 2nd ed., Addison-Wesley Publishing Company, Reading, MA, 1994.

[11] R. P. Grimaldi, Fibonacci and Catalan Numbers, John Wiley \& Sons, Inc., Hoboken, NJ, 2012; available online at https://doi.org/10.1002/9781118159743

[12] B.-N. Guo and F. Qi, On the Wallis formula, Internat. J. Anal. Appl. 8 (2015), no. 1, 30-38.

[13] P. Hilton and J. Pedersen, Catalan numbers, their generalization, and their uses, Math. Intelligencer 13 (1991), no. 2, 64-75; available online at https://doi.org/10.1007/BF03024089

[14] C. Jordan, Calculus of Finite Differences, Hungarian Agent Eggenberger Book-Shop, Budapest, 1939.

[15] D. A. Klarner, Correspondences between plane trees and binary sequences, J. Combin. Theory 9 (1970), no. 4, 401-411; available online at https://doi.org/10.1016/S0021-9800(70)80093-X

[16] T. Koshy, Catalan Numbers with Applications, Oxford University Press, Oxford, 2009.

[17] P. Larcombe, On the history of the Catalan numbers: a first record in China, Math. Today (Southend-on-Sea) 35 (1999), no. 3, 89-89.

[18] P. J. Larcombe, The 18th century Chinese discovery of the Catalan numbers, Math. Spectrum 32 (1999/2000) no. $1,5-7$.

[19] W.-H. Li and F. Qi, A further generalization of the Catalan numbers and its explicit formula and integral representation, Authorea Preprints (2020), available online at https://doi.org/10.22541/au.159844115. 58373405

[20] F.-F. Liu, X.-T. Shi, and F. Qi, A logarithmically completely monotonic function involving the gamma function and originating from the Catalan numbers and function, Glob. J. Math. Anal. 3 (2015), no. 4, 140-144; available online at https://doi.org/10.14419/gjma.v3i4.5187.

[21] X. R. Ma, The general solution of Ming Antu's problem, Acta Math. Sin. (Engl. Ser.) 20 (2004), no. 1, 157-162; available online at https://doi.org/10.1007/s10114-003-0282-2

[22] M. McKiernan, On the nth derivative of composite functions, Amer. Math. Monthly 63 (1956), no. 5, 331-333; available online at https://doi.org/10.2307/2310518

[23] M. Mahmoud and F. Qi, Three identities of the Catalan-Qi numbers, Mathematics 4 (2016), no. 2, Article 35, 7 pages; available online at https://doi.org/10.3390/math4020035

[24] A. Nkwanta and A. Tefera, Curious relations and identities involving the Catalan generating function and numbers, J. Integer Seq. 16 (2013), no. 9, Article 13.9.5, 15 pages.

[25] F. W. J. Olver, D. W. Lozier, R. F. Boisvert, and C. W. Clark (eds.), NIST Handbook of Mathematical Functions, Cambridge University Press, New York, 2010; available online at http://dlmf.nist.gov/ 
[26] K. A. Penson and J.-M. Sixdeniers, Integral representations of Catalan and related numbers, J. Integer Seq. 4 (2001), no. 2, Article 01.2.5.

[27] F. Qi, An improper integral, the beta function, the Wallis ratio, and the Catalan numbers, Probl. Anal. Issues Anal. 7 (25) (2018), no. 1, 104-115; available online at https://doi.org/10.15393/j3.art.2018.4370

[28] F. Qi, Inverse of a triangular matrix and several identities of Catalan numbers, J. Hunan Inst. Sci. Technol. (Nat. Sci.) 33 (2020), no. 2, 1-11 and 22; available online at https://doi.org/10.16740/j.cnki.cn43-1421/ n.2020.02.001 (Chinese)

[29] F. Qi, Parametric integrals, the Catalan numbers, and the beta function, Elem. Math. 72 (2017), no. 3, 103-110; available online at https://doi.org/10.4171/EM/332

[30] F. Qi, Some properties of the Catalan numbers, Ars Combin. (2022), in press; available online at https: //www.researchgate.net/publication/328891537

[31] F. Qi, A. Akkurt, and H. Yildirim, Catalan numbers, k-gamma and k-beta functions, and parametric integrals, J. Comput. Anal. Appl. 25 (2018), no. 6, 1036-1042.

[32] F. Qi and P. Cerone, Some properties of the Fuss-Catalan numbers, Mathematics 6 (2018), no. 12, Article 277, 12 pages; available online at https://doi.org/10.3390/math6120277

[33] F. Qi and B.-N. Guo, From inequalities involving exponential functions and sums to logarithmically complete monotonicity of ratios of gamma functions, J. Math. Anal. Appl. 493 (2021), no. 1, Art. 124478, 19 pages; available online at https://doi.org/10.1016/j.jmaa.2020.124478

[34] F. Qi and B.-N. Guo, Integral representations of the Catalan numbers and their applications, Mathematics 5 (2017), no. 3, Article 40, 31 pages; available online at https://doi.org/10.3390/math5030040

[35] F. Qi and B.-N. Guo, Logarithmically complete monotonicity of a function related to the Catalan-Qi function, Acta Univ. Sapientiae Math. 8 (2016), no. 1, 93-102; available online at https://doi.org/10.1515/ ausm-2016-0006

[36] F. Qi and B.-N. Guo, Logarithmically complete monotonicity of Catalan-Qi function related to Catalan numbers, Cogent Math. 3 (2016), Paper No. 1179379, 6 pages; available online at https://doi.org/10. $1080 / 23311835.2016 .1179379$

[37] F. Qi and B.-N. Guo, Some properties and generalizations of the Catalan, Fuss, and Fuss-Catalan numbers, Chapter 5 in Mathematical Analysis and Applications: Selected Topics, pp. 101-133; Edited by Michael Ruzhansky, Hemen Dutta, and Ravi P. Agarwal; Published by John Wiley \& Sons, Inc. 2018; available online at https://doi.org/10.1002/9781119414421.ch5

[38] F. Qi and B.-N. Guo, Sums of infinite power series whose coefficients involve products of the Catalan-Qi numbers, Montes Taurus J. Pure Appl. Math. 1 (2019), no. 2, Art. ID MTJPAM-D-19-00007, 1-12

[39] F. Qi, W.-H. Li, J. Cao, D.-W. Niu, and J.-L. Zhao, An analytic generalization of the Catalan numbers and its integral representation, arXiv preprint (2020), available online at https://arxiv.org/abs/2005.13515v1

[40] F. Qi, M. Mahmoud, X.-T. Shi, and F.-F. Liu, Some properties of the Catalan-Qi function related to the Catalan numbers, SpringerPlus 5 (2016), Paper No. 1126, 20 pages; available online at https://doi.org/ 10.1186/s40064-016-2793-1.

[41] F. Qi, D.-W. Niu, D. Lim, and B.-N. Guo, Closed formulas and identities for the Bell polynomials and falling factorials, Contrib. Discrete Math. 15 (2020), no. 1, 163-174; available online at https://doi.org/ $10.11575 / \mathrm{cdm} . \mathrm{v} 15 \mathrm{i1} .68111$

[42] F. Qi, D.-W. Niu, D. Lim, and Y.-H. Yao, Special values of the Bell polynomials of the second kind for some sequences and functions, J. Math. Anal. Appl. 491 (2020), no. 2, Art. 124382, 31 pages; available online at https://doi.org/10.1016/j.jmaa.2020.124382

[43] F. Qi, X.-T. Shi, and P. Cerone, A unified generalization of the Catalan, Fuss, and Fuss-Catalan numbers, Math. Comput. Appl. 24 (2019), no. 2, Art. 49, 16 pages; available online at https://doi.org/10.3390/ mca24020049.

[44] F. Qi, X.-T. Shi, and F.-F. Liu, An integral representation, complete monotonicity, and inequalities of the Catalan numbers, Filomat 32 (2018), no. 2, 575-587; available online at https://doi.org/10.2298/ FIL1802575Q

[45] F. Qi, X.-T. Shi, F.-F. Liu, and D. V. Kruchinin, Several formulas for special values of the Bell polynomials of the second kind and applications, J. Appl. Anal. Comput. 7 (2017), no. 3, 857-871; available online at https://doi.org/10.11948/2017054

[46] F. Qi, X.-T. Shi, M. Mahmoud, and F.-F. Liu, Schur-convexity of the Catalan-Qi function related to the Catalan numbers, Tbilisi Math. J. 9 (2016), no. 2, 141-150; available online at https://doi.org/10.1515/ tmj-2016-0026

[47] F. Qi, X.-T. Shi, M. Mahmoud, and F.-F. Liu, The Catalan numbers: a generalization, an exponential representation, and some properties, J. Comput. Anal. Appl. 23 (2017), no. 5, 937-944. 
[48] F. Qi and Y.-H. Yao, Simplifying coefficients in differential equations for generating function of Catalan numbers, J. Taibah Univ. Sci. 13 (2019), no. 1, 947-950; available online at https://doi.org/10.1080/ 16583655.2019 .1663782

[49] F. Qi, Q. Zou, and B.-N. Guo, The inverse of a triangular matrix and several identities of the Catalan numbers, Appl. Anal. Discrete Math. 13 (2019), no. 2, 518-541; available online at https://doi.org/10. 2298/AADM190118018Q

[50] S. Roman, An Introduction to Catalan Numbers, with a foreword by Richard Stanley, Compact Textbook in Mathematics, Birkhäuser/Springer, Cham, 2015; available online at https://doi.org/10.1007/ 978-3-319-22144-1

[51] X.-T. Shi, F.-F. Liu, and F. Qi, An integral representation of the Catalan numbers, Glob. J. Math. Anal. 3 (2015), no. 3, 130-133; available online at https://doi.org/10.14419/gjma.v3i3.5055

[52] M. K. Soni, A. Soni, and D. Bansal, Some geometric properties of analytic series whose coefficients are reciprocal of Fuss-Catalan numbers, Electron. J. Math. Anal. Appl. 6 (2018), no. 2, 246-254.

[53] M. Z. Spivey, The Art of Proving Binomial Identities, Discrete Mathematics and its Applications (Boca Raton), CRC Press, Boca Raton, FL, 2019; available online at https://doi.org/10.1201/9781351215824

[54] R. P. Stanley, Catalan Numbers, Cambridge University Press, New York, 2015; available online at https: //doi.org/10.1017/CB09781139871495

[55] N. M. Temme, Special Functions: An Introduction to Classical Functions of Mathematical Physics, A WileyInterscience Publication, John Wiley \& Sons, Inc., New York, 1996; available online at https://doi.org/ $10.1002 / 9781118032572$

[56] C. I. Vălean, (Almost) Impossible Integrals, Sums, and Series, with a foreword by Paul J. Nahin, Problem Books in Mathematics, Springer, Cham, 2019; available online at https://doi.org/10.1007/ 978-3-030-02462-8

[57] L. Yin and F. Qi, Several series identities involving the Catalan numbers, Trans. A. Razmadze Math. Inst. 172 (2018), no. 3, 466-474; available online at https://doi.org/10.1016/j.trmi.2018.07.001.

Department of Fundamental Courses, Zhengzhou University of Science and Technology, Zhengzhou 450064, Henan, CHINA

Email address: wen.hui.li102@gmail.com, wen.hui.li@foxmail.com

$U R L$ : https://orcid.org/0000-0002-1848-8855

Institute of Mathematics, Henan Polytechnic University, Jiaozuo 454010, Henan, China; College of Mathematics and Physics, Inner Mongolia University for Nationalities, Tongliao 028043, Inner Mongolia, China; School of Mathematical Sciences, Tianjin Polytechnic University, Tianjin 300387, CHINA

Email address: qifeng618@gmail.com, qifeng618@hotmail.com, qifeng618@qq.com

$U R L$ : https://qifeng618.wordpress.com, https://orcid.org/0000-0001-6239-2968

Department of Mathematics, Higher Institute for Applied Sciences and Technology, P.O. Box 31983, Damascus, SyRIA

Email address: omran_kouba@hiast.edu.sy, kouba.omran@gmail.com

$U R L$ : https://orcid.org/0000-0002-9577-3326

Department of Mathematics, School of Arts and Sciences, International University of Beirut, Lebanese International University, Beirut, Lebanon

Email address: issam.kaddoura@liu.edu.lb 